\title{
Fractional properties of geophysical field variability on the ex- ample of hydrochemical parameters
}

\author{
Boris Shevtsov ${ }^{1, \star}$ and Olga Shevtsova ${ }^{2, \star \star}$ \\ ${ }^{1}$ Institute of Cosmophysical Research and Radio Wave Propagation FEB RAS (IKIR FEB RAS), \\ 684034, Mirnaya str., 7, Paratunka, Elizovskiy district, Kamchatka, Russia \\ ${ }^{2}$ V.I. Il'ichev Pacific Oceanological Institute FEB RAS, Vladivostok, Russia
}

\begin{abstract}
Using the properties of compound Poisson process and its fractional generalizations, statistical models of geophysical fields variability are considered on an example of hydrochemical parameters system. These models are universal to describe objects of different nature and allow us to explain various pulsing regime. Manifestations of non-conservatism in hydrochemical parameters system and the advantages of the system approach in the description of geophysical fields variability are discussed.
\end{abstract}

Universal character and wide use of power laws in nature are associated with the fundamental summation of identically distributed random variables and the law of large numbers. We can list many examples of power law characterizing complicated systems of heterogeneous elements in different areas of natural sciences (astrophysics, seismology, turbulence theory etc.) including the same relations describing sea water chemical parameters system $[1,2]$. The elements of this system are interacting between each other (directly and indirectly) and with the elements of other systems represented by hydrophysical fields and hydrobiology objects. All these interactions create a great diversity of variability factors affecting the chemical system behavior.

Fig. 1 represents the dependencies of standards $\sigma$ on time average concentrations $\bar{\rho}$ of 16 chemical parameters measured at 13 points of diurnal observations in Amur Bay of the Sea of Japan (for initial data see [3]). Two regions are notable in the concentration range of $10^{-4}-3.3 \times 10^{4} \mathrm{mg} / \mathrm{kg}$, they are: with $\bar{\rho}<10^{2} \mathrm{mg} / \mathrm{kg}$ for non-conservative parameters and with $\bar{\rho}>10^{2} \mathrm{mg} / \mathrm{kg}$ for conservative ones. All the data of non-conservative parameters (dissolved oxygen, nutrients, carbonic parameters) are approximated by a unified power law regression with the exponent $\gamma=0.68 \pm 0.02$. The conservative parameter data (salinity and components of major salt composition) are approximated by a series of separate parallel regressions with $\gamma=1$, each of which refers to a separate observation point.

It is obvious that for each concentration region there is a own set of peculiar basic factors of variability. In the parameters system under consideration, the fluctuation regime change corresponds to the boundary concentration of $\bar{\rho}=10^{2} \mathrm{mg} / \mathrm{kg}$. In this paper, we consider how and what factors determine the behavior of a hydrochemical system.

Before we turn to the discussion of a statistical model of hydrochemical parameters system behavior, we shall adduce some analogies. For example, a flow of seismic events may be considered

\footnotetext{
^e-mail: bshev@ikir.ru

$\star \star$ e-mail: OV_shevtsova@mail.ru
} 
as a system of heterogeneous elements which differ by energy and spatial scales and the well-known Gutenberg-Richter law is analogous to the power law illustrated in Fig. 1. Cosmic ray intensity distribution depending on their energies and turbulence spectrum are also presented by power laws. In all these cases dependence slopes characterize the system behavior on the whole.

Variability of hydrochemical parameters concentrations $\rho_{i}(x, t)$ is determined by their standards $\sigma_{i}(x, t)$, where $i=1, \ldots, N$ is a parameter number. We consider the dependence $\sigma_{i}(x, t)$ on average values of parameter concentrations $\bar{\rho}_{i}(x, t)$. In our statistical model of hydrochemical parameter system behavior, we take into account the main factors of variability responsible for parameters fluctuations. In this paper we consider a case when a sum $\rho_{i}(x, t)=\sum_{k=0}^{n(t)} \Delta \rho_{i}\left(x, t_{k}\right)$ of independent random increments $\Delta \rho_{i}\left(x, t_{k}\right)$ with distribution $p\left(\Delta \rho_{i}\right)$ is a compound (generalized) Poisson process. To take into account the memory effects of time increment dependence, we consider fractional compound Poisson process $[4,5]$.

When we impose the condition $\Delta \rho_{i} \geq 0$, we obtain an additive, positively directed process which describes substance concentration increase in an examination volume. To estimate the loss of substance concentration in a volume, it is necessary to introduce an alternating process, i.e. instead of the condition $\Delta \rho_{i} \geq 0$ we assume that $\rho_{i}(x, t) \geq 0$.

The impact from turbulent fluctuations is formed by volume split and their random mixing. That results in a power law spectrum of fluctuations which is applied in the discussion of additive statistic model based on Poisson process.

To describe sea water tidal oscillations of we consider the changes of $\Delta \rho_{i}(x, t)$, proportional to the gradient of $\rho_{i}(x, t)$ value and current speed. Correlation in tidal oscillations may be considered as memory effect which may be taken into account by fractional compound Poisson process.

For a standard compound Poisson process $\rho_{i}(x, t)=\sum_{k=0}^{n(t)} \Delta \rho_{i}\left(x, t_{k}\right)$ with flux vof independent random changes $\Delta \rho_{i}\left(x, t_{k}\right)$ at independent random time moments $t_{k}$ (omitting the variables $i$ and $x$ ) we have:

$$
\begin{gathered}
\bar{\rho}(t)=\overline{\Delta \rho} \bar{n}(t), \quad \bar{n}(t)=v t, \quad \overline{\rho^{2}}(t)=\overline{\Delta \rho^{2}} \bar{n}(t)+\overline{\Delta \rho}^{2}\left(\overline{n^{2}}(t)-\bar{n}(t)\right), \\
\overline{n^{2}}(t)=\bar{n}(t)+\bar{n}^{2}(t), \quad \sigma^{2}(t)=\overline{\Delta \rho^{2}} \bar{n}(t), \quad \sigma(t)=\sqrt{\overline{\Delta \rho^{2}}} \sqrt{\bar{n}(t)} .
\end{gathered}
$$

The relation (2) results in a power law of chemical parameters variability with their average values with the exponent of 0.5. For relative variability $\sigma(t) / \bar{n}(t)=\sqrt{\overline{\Delta \rho^{2}}} / \sqrt{\bar{n}(t)}$ the exponent is -0.5 .

Turbulence forms power law fluctuations which may have not the value $\overline{\Delta \rho^{2}}$. For this case, instead of the dispersion $\overline{\Delta \rho^{2}}$ we consider its fractional analogue:

$$
\sigma^{\alpha}(t)=\overline{\Delta \rho^{\alpha}} \bar{n}(t), \quad \sigma(t)=\left(\overline{\Delta \rho^{\alpha}} \bar{n}(t)\right)^{\gamma}, \quad 1 \leq \alpha \leq 2, \quad 0.5 \leq \gamma=1 / \alpha \leq 1 .
$$

The obtained power law (3) agrees well with the experimental regression in Fig. 1 in region $\bar{\rho}<10^{2} \mathrm{mg} / \mathrm{kg}$ which has the exponent $\gamma=0.68$. This value can be considered as a characteristic of non-conservative hydrochemical parameters system behavior on the whole. The physical meanings of the values $\alpha$ and $\gamma=1 / \alpha$ are turbulence fluctuation parameters in a system. When $\alpha=1.5$, we have a reverse value of $\gamma=1 / 1.5=2 / 3$. A value, close to this one, appear in many natural power laws.

To explain the break (cut off) of a power law in the region of boundary concentration $\bar{\rho}=10^{2} \mathrm{mg} / \mathrm{kg}$, we should take into account the disturbance of random event independence during concentration change. The tidal gradient mechanism of fluctuations with their long correlations on the semi-diurnal period may be considered as a cause of this disturbance. 


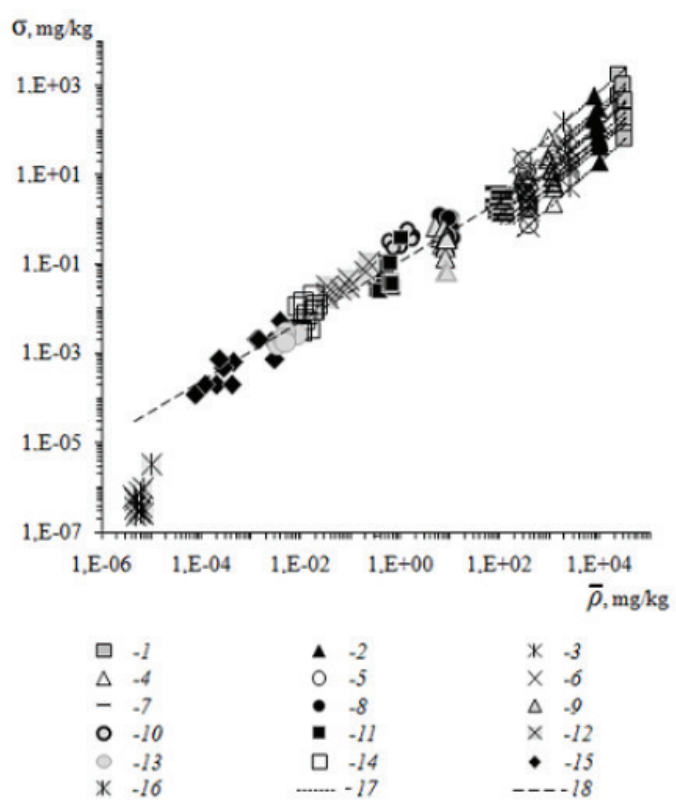

Figure 1. The relationship between the average concentration $\bar{\rho}$ and the standard $\sigma$ for a complex of hydrochemical parameters at 13 horizons of four daily stations in various parts of Amur Bay. $1-16$ - salinity, $\mathrm{Na}^{+}, \mathrm{SO}_{4}^{2-}$, $\mathrm{Mg}^{2+}, \mathrm{K}^{+}, \mathrm{Ca}^{2+}, \mathrm{HCO}_{3}^{-}, \mathrm{CO}_{3}^{2-}, \mathrm{O}_{2}, \mathrm{SiO}_{3}, \mathrm{CO}_{2}, \mathrm{NH}_{4}^{+}, \mathrm{NO}_{3}^{-}, \mathrm{PO}_{4}^{3-}, \mathrm{NO}_{2}^{-}, a_{\mathrm{H}^{+}}$, respectively. 17 and 18 - power law regression for the

In Fig. 1 the fluctuation tidal mechanism manifests in the form of almost vertical grouping of data of salinity and components of major salt composition in the region of $\bar{\rho}>10^{2} \mathrm{mg} / \mathrm{kg}$. Here the tidal mechanism prevails over the turbulent fluctuation one. As concentration decreases, the vertical grouping of data vanishes. The dependence of increments $\Delta \rho_{i}(x, t)$ is violated that results in the break of the power law.

In order to obtain a statistical model of fluctuations with memory, as an example, we consider the fractal Poisson process [4], for which we have:

$$
\sigma_{\mu}^{2}(t)=\left[\bar{n}_{\mu}(t)+\bar{n}_{\mu}^{2}(t)\left(\frac{\mu \Gamma(\mu) \Gamma(1 / 2)}{\Gamma(\mu+1 / 2)}-1\right)\right], \quad \bar{n}_{\mu}(t)=\frac{v t^{\mu}}{\Gamma(\mu+1)},
$$

where $\Gamma(\mu)$ is a gamma-function, $\mu$ is a fractional parameter of memory of fluctuations, $0<\mu \leq 1$. When $\mu=1$, the fractional process transforms into a usual one. The second turn in square brackets is responsible for the effects of memory. When $\mu=1$, this turn is zero.

The fractional Poisson process has distribution for waiting time different from an exponential curve. Thus, fluctuations cannot be considered independent.

The fractional Poisson process differs from a standard one by power law distribution of waiting time for which long fading is possible.

When the substances concentration is low, the fractional Poisson process behaves as a standard one. When the concentration is high, it turns to the asymptotic $\sigma(t) \simeq \bar{n}_{\mu}(t)$ with the exponent 1 . This is just the reason of the break (cut off) which is associated with memory effects. 
For the fractional compound Poisson process [5] we have:

$$
\sigma_{\mu}^{2}(t)=\left[\overline{\Delta \rho^{2}} \bar{n}_{\mu}(t)+\overline{\Delta \rho}^{2} \bar{n}_{\mu}^{2}(t) g(\mu)\right], \quad g(\mu)=\frac{\mu \Gamma(\mu) \Gamma(1 / 2)}{\Gamma(\mu+1 / 2)}-1 .
$$

It is clear that the break point also depends on the moments of fluctuations $\overline{\Delta \rho^{2}}$ and $\overline{\Delta \rho}^{2}$. For high concentrations, we obtain the asymptotic $\sigma(t) \simeq \overline{\Delta \rho} g(\mu)^{1 / 2} \bar{n}_{\mu}(t)$. In Fig. 1 for high concentrations of $\bar{\rho}>10^{2} \mathrm{mg} / \mathrm{kg}$, a set of parallel lines for different observation points corresponds to this asymptotic.

For the fractional compound Poisson process, which has a power law for waiting time, we have:

$$
\sigma_{\mu}^{\alpha}(t)=\left[\overline{\Delta \rho^{\alpha}} \bar{n}_{\mu}(t)+\overline{\Delta \rho}^{\alpha} \bar{n}_{\mu}^{\alpha}(t) g(\mu)\right] .
$$

When the concentration is low, this relation is analogous to (3). When the concentration is high, we have the same asymptotic as for (5).

Instead of the fractional one, we can take other generalization of the Poisson process with memory effects. In this case, the results which were presented above change slightly.

Finally, we discuss the influence of the variability factors associated with non-conservative behavior of hydrochemical parameters. These are chemical, physical-chemical and biochemical reactions which cause smoothing or intensification of turbulent fluctuation manifestations. As a result, experimental data may deviate from the power law regression to any extent. The complex of these deviations may be considered as a characteristic of non-conservative behavior of a hydrochemical parameters system.

\section{Conclusions}

1. Based on the most general assumptions on the fluctuation nature in the system of hydrochemical parameters, a model of power law of their variability on average concentration was obtained.

2. Power law break (cut off) was explained as a manifestation of variability tidal mechanism memory effect.

3. Features of gradient variability in power law were detected in the form of data vertical grouping.

4. The role of the non-conservative behavior of the hydrochemical parameters in the deviations of their variability on power law was shown.

\section{References}

[1] O.V. Shevtsova, Increase in the relative diurnal variability of hydrochemical indices in Amur Bay, Sea of Japan, in Doklady Earth Sciences (Springer Science \& Business Media, 2015), Vol. 460, p. 73

[2] O.V. Shevtsova, Water Resources 40, 181 (2013)

[3] O.V. Shevtsova, Water Resources 28, 666 (2001)

[4] D.O. Cahoy, V.V. Uchaikin, W.A. Woyczynski, Journal of Statistical Planning and Inference 140, 3106 (2010)

[5] N. Laskin, Communications in Nonlinear Science and Numerical Simulation 8, 201 (2003) 